\title{
Measuring Mass and Spin of Dark Matter Particles with the Aid Energy Spectra of Single Lepton and Dijet at the $\mathrm{e}^{+} \mathrm{e}^{-}$ Linear Collider
}

\author{
I. F. Ginzburg1,2 \\ ${ }^{1}$ Sobolev Institute of Mathematics, Novosibirsk, Russia \\ ${ }^{2}$ Novosibirsk State University, Novosibirsk, Russia \\ Email: ginzburg@math.nsc.ru
}

Received 18 January 2014; revised 18 February 2014; accepted 16 March 2014

Copyright (C) 2014 by author and Scientific Research Publishing Inc.

This work is licensed under the Creative Commons Attribution International License (CC BY). http://creativecommons.org/licenses/by/4.0/

\section{c) (i) Open Access}

\section{Abstract}

In many models stability of Dark Matter particles $D$ is ensured by conservation of a new quantum number referred to as $D$-parity. Our models also contain charged $D$-odd particles $D^{ \pm}$ with the same spin as $D$. Here we propose a method to precisely measure the masses and spins of $D$-particles in the process $\mathrm{e}^{+} \mathrm{e}^{-} \rightarrow D^{+} D^{-} \rightarrow D D W^{+} W^{-} \rightarrow D D W(q \bar{q})(\ell v)$ with a signature dijet $+\mu($ or $e)+$ nothing. It is shown that the energy distribution of the lepton has singular points (upper edge and kinks or a peak) whose positions are kinematically determined. For precise determination of $D$ and $D^{ \pm}$masses, it is sufficient to measure these singular points and upper edge of dijet energy spectrum. After this procedure, even an approximate measurement of the corresponding cross section allows a determination of the spin of $D$ particles to be performed. New points of this work are: 1 ) We propose to use only the well measurable energy spectra of individual leptons and the upper edge of the dijet energy spectrum. 2) We propose to identify the spin of $D$-particles via value of the cross section for the discussed process.

\section{Keywords}

Dark Matter, W-Boson, Lepton, Linear Collider 


\section{Introduction}

\subsection{Models}

In the broad class of models Dark Matter (DM) consists of particles $D$ similar to those in SM, with the following properties:

- The neutral DM particle $D$ with mass $M_{D}$ and spin $s_{D}=0$ or $1 / 2$ has a new conserved quantum number, which we call the $D$-parity. All known particles are $D$-even, while the $D$ is $D$-odd.

- In addition to $D$, other $D$-odd particles exist: a charged $D^{ \pm}$and (sometimes) a neutral $D^{A}$, with the same spin $s_{D}$ and with masses $M_{+}, M_{A}>M_{D}$. The $D$-parity conservation ensures stability of the lightest $D$-odd particle $D$.

- These $D$-particles interact with the SM particles via the Higgs boson $D D h, D^{+} D^{-} h, D^{A} D^{A} h$ and via the covariant derivative in the kinetic term of the Lagrangian. These are the gauge interactions $D^{+} D^{-} \gamma$, $D^{+} D^{-} Z, D^{+} D W^{-}, D^{+} D^{A} W^{-}, D^{A} D Z$ with the standard electroweak couplings $g, g^{\prime}$ and e (coupling to $Z$ can be added by a mixing factor $\mu_{M} \leq 1$, deviation from 1 appears due to possible mixing of $D$ with other $D$-odd neutrals).

- The first example of such model provides well known MSSM (see e.g. [1]-[6]) for specific set of parameters. Here our term $D$-parity means $R$-parity. For the considered set of parameters, $D$ is the lightest neutralino $\chi_{1}^{0}$, the heavier neutralino $\chi_{2}^{0}$ can play role $D^{A}$ and the next in mass $D$-odd particle is the lightest chargino, spin of these $D$-particles $s_{D}=1 / 2$. The other $D$-odd particles (in particular, sleptons and squarks) are supposed to be heavier than the ILC beam energy $E$.

- The second example of such models provides the Inert Doublet Model (IDM) (see e.g. [7]-[13] and Appendix A). That is the $Z_{2}$ symmetric Two Higgs Doublet Model, containing two scalar doublets $\phi_{S}$ and $\phi_{D}$. The "standard" scalar (Higgs) doublet $\phi_{S}$ is responsible for electroweak symmetry breaking and the masses of fermions and gauge bosons just as in the Standard Model (SM). The second scalar doublet $\phi_{D}$ doesn't receive vacuum expectation value and doesn't couple to fermions. In this model the $D$-parity conservation is ensured by a $Z_{2}$ symmetry, four degrees of freedom of the Higgs doublet $\phi_{S}$ are the same as in the SM: three Goldstone modes and the standard Higgs boson $h$. All the components of the scalar doublet $\phi_{D}$ are realized as the massive $D$-particles: two charged $D^{ \pm}$and two neutral ones $D, D^{A}$ with masses $M_{+}$, $M_{D}, M_{A}$ respectively with $M_{+}, M_{A}>M_{D}$. IDM contains no other $D$-odd particles. All $D$-particles have spin $s_{D}=0$.

A possible value of mass $M_{D}$ is limited by stability of $D$ during the Universe existence [14]-[20]. The non-observation of the processes $\mathrm{e}^{+} \mathrm{e}^{-} \rightarrow D^{+} D^{-}$and $\mathrm{e}^{+} \mathrm{e}^{-} \rightarrow D D^{A}$ at LEP gives $M_{+}>90 \mathrm{GeV}$ and $M_{A}>100 \mathrm{GeV}$ (at $M_{A}-M_{D}>10 \mathrm{GeV}$ ) [11]-[13]. Limitations for masses of neutralino and chargino can be found in [20]. For IDM, limitations for parameters of $Z$-peak, $S$ and $T$ results in ([11]-[13] [20])

$$
|\Delta T|=2.16\left(\frac{M_{+}-M_{D}}{\mathrm{v}}\right) \cdot\left|\frac{M_{+}-M_{A}}{\mathrm{v}}\right|<0.15
$$

with $\mathrm{v}=246 \mathrm{GeV}$ expectation value of Higgs field. Further, we will have in mind $M_{D} \lesssim 80 \mathrm{GeV}$ and assume $M_{+}-M_{D}, M_{A}-M_{D}>20 \mathrm{GeV}$.

\subsection{The Problem}

The neutral and stable $D$ can be produced and detected via production $D^{ \pm}$or $D^{A}$ and subsequent decay $D^{ \pm} \rightarrow D W^{ \pm}, D^{A} \rightarrow D Z$ with either on shell (real) or off shell $W^{ \pm}$or $Z$. The off shell $W$ emerges as a $q \bar{q}$ pair (dijet ${ }^{1}$ ) or $\ell v$, having the same quantum numbers as $W$ but with an effective mass $M^{*}<M_{W}$. From now on, $W$ or $Z$ refers to any of these two cases.

To discover the DM particle, one needs to specify such processes with a clear signature. The $\mathrm{e}^{+} \mathrm{e}^{-}$Collider ILC/CLIC provides an excellent opportunity for this task (see, e.g., [21] [22]) in the process $\mathrm{e}^{+} \mathrm{e}^{-} \rightarrow D^{+} D^{-}$ with a clear signature, see Equations (6) and (7) below. The cross section of this process is a large fraction of the total cross section of $\mathrm{e}^{+} \mathrm{e}^{-}$annihilation, Section 3.3.

The masses $M_{+}$and $M_{D}$ could be found via the edges of the energy distribution of dijets, originating from $W$ from decay $D^{ \pm} \rightarrow D W^{ \pm}$, section 2.2, 2.4 (see [5] [6] for MSSM and [11]-[13] for IDM). However, this method cannot provide a good accuracy in measuring the mass. Indeed, the individual jet energy measurement ${ }^{1}$ We use term "dijet” for all products of $W$ decay in $q \bar{q}$ mode that are 2 quark jets or 2 quark jets plus gluon jet(s) or few hadrons for off shell $W$ with small effective mass. 
suffers from a sizable uncertainty. In particular, this uncertainty smoothes the lower edge in the dijet energy spectrum.

On the contrary, the lepton energy can be measured much more precisely. In this paper we show, first, that the energy distribution of leptons has singular points whose positions are kinematically determined. Measuring positions of these singularities will allow, in principle, to determine masses $M_{D}$ and $M_{+}$with good precision (Sections 2.3 and 2.4). In contrast to [5]-[6] [11]-[13], our description is suitable for different models.

Moreover, we present a simple method for measuring spin of DM particles in these very experiments.

The discussed problem differs strongly from that for the case when the lightest charged D-odd particle is slepton (another set of parameters of MSSM). In the latter case DM particles are produced via slepton pair $\mathrm{e}^{+} \mathrm{e}^{-} \rightarrow \tilde{\ell}^{+} \tilde{\ell}^{-} \rightarrow \ell^{+} \ell^{-} \chi_{0} \chi_{0}$. First of all, signature of this process is quite different from that one in our problem (6), (7). Second, the energy of observable lepton decay product of slepton is measurable well in each individual event, in difference with our case, when similar product of decay, $W$, is seen as dijet or lepton plus neutrino with badly measurable energy in each individual event. Therefore, the approach used in the analysis of slepton production (cf. [23]-[25]) cannot be applied directly to our problem.

The overall picture is summarized in Section 3. Short conclusion is given in Section 4.

In the Appendix B we discuss the potential of the process $\mathrm{e}^{+} \mathrm{e}^{-} \rightarrow D D^{A} \rightarrow D D Z$ for similar problems, for completeness. In contrast with previous studies, we find that this potential is not too high.

In the Appendix $\mathbf{C}$ we consider possible background processes and show that the most of them can be neglected at the analysis.

\subsection{Scale of Cross Sections}

We express discussed cross sections via

$$
\sigma_{0} \equiv \sigma\left(\mathrm{e}^{+} \mathrm{e}^{-} \rightarrow \gamma \rightarrow \mu^{+} \mu^{-}\right)=4 \pi \alpha^{2} / 3 s .
$$

The total cross section of $\mathrm{e}^{+} \mathrm{e}^{-}$annihilation at ILC for $\sqrt{\mathrm{s}} \equiv 2 E>200 \mathrm{GeV}$ is $\sim 10 \sigma_{0}$. The annual integrated luminosity $L$ for the ILC project [22] gives

$$
L \sigma_{0} \sim 3 \times 10^{5} .
$$

The process $\mathrm{e}^{+} \mathrm{e}^{-} \rightarrow D^{+} D^{-}$represents a significant fraction of all $\mathrm{e}^{+} \mathrm{e}^{-}$annihilation events-see (19), (20), Figure 2 and Table 2. With the luminosity (3), the annual number of events of discussed type will be $(0.6 \div 3) \times 10^{5}$, depending on $M_{+} / E$ and $s_{D}$, and about $1 / 3$ of them (in the mode with $\mathrm{e}$ or $\mu$ plus dijet) are suitable for our analysis.

\section{The Process $\mathrm{e}^{+} \mathrm{e}^{-} \rightarrow D^{+} D^{-}$}

Note before all that the energies, $\gamma$-factors and velocities of $D^{ \pm}$are

$$
E_{ \pm}=E=\sqrt{s} / 2, \gamma_{ \pm}=E / M_{+}, \quad \beta_{ \pm}=\sqrt{1-M_{+}^{2} / E^{2}} .
$$

\subsection{The Signature}

- If $M_{A}>M_{+}$or $D^{A}$ is absent, once produced, particles $D^{ \pm}$decay fast (with a unit probability) to $D W^{ \pm}$,

$$
\mathrm{e}^{+} \mathrm{e}^{-} \rightarrow D^{+} D^{-} \rightarrow D D W^{+} W^{-} .
$$

The observable states are decay products of $W$ with a large missing transverse energy $Z_{T}$ carried away by the invisible $D$-particle, and the missing mass of particles escaping observation $M\left(Z_{T}\right)$ is large. In contrast to the LHC, where a large flux of low $p_{\perp}$ particles demands an additional $p_{\perp}$ cut off, at $\mathrm{e}^{+} \mathrm{e}^{-}$LC such particles are absent.

Therefore, the signatures of the process in the modes suitable for observation are

$$
\begin{aligned}
& \mathrm{e}^{+} \mathrm{e}^{-} \rightarrow D D(W \rightarrow q \bar{q})(W \rightarrow q \bar{q}): \text { Two dijets + nothing, } \\
& \text { with energy of each dijet }<E \text {, with large } E_{T} \text { and large } M\left(E_{T}\right) .
\end{aligned}
$$




$$
\begin{aligned}
& \mathrm{e}^{+} \mathrm{e}^{-} \rightarrow D D(W \rightarrow \ell v)(W \rightarrow q \bar{q}): \text { One dijet }+ \text { e or } \mu+\text { nothing, } \\
& \text { with energy of each dijet or lepton }<E \text {, with large } E_{T}^{\prime} \text { and large } M\left(E_{T}\right) .
\end{aligned}
$$

At $M^{*}>5 \mathrm{GeV}$, the branching ratios for different channels of $W$ decay are roughly identical for on-shell $W$ [20] and off-shell $W$. In particular, the fraction of events with signature $(6 a)$ is $0.676^{2} \approx 0.45$. The fraction of events with signature $(6 b)$ is $2 \times 0.676 \times 2 \times(1+0.17) \times 0.108 \approx 0.33$ (here 0.17 is a fraction of $\mu$ or e from the decay of $\tau)$. At $M^{*}<5 \mathrm{GeV}, B R(\mathrm{e} v)$ and $B R(\mu v)$ increase, while the dijet becomes a set of a few hadrons.

- If $\boldsymbol{M}_{+}>\boldsymbol{M}_{A}$, when analysing the main process $\mathrm{e}^{+} \mathrm{e}^{-} \rightarrow D^{+} D^{-}$, one more decay channel is added, $D^{ \pm} \rightarrow D^{A} W^{ \pm} \rightarrow D Z W^{ \pm}$. Its branching ratio $B=B R\left(D^{+} \rightarrow D^{A} W^{+}\right)$is typically less than 0.5 (see discussion in section 2.4). Particle $D^{A}$ decays fast to $D Z$, creating new cascades

$\mathrm{e}^{+} \mathrm{e}^{-} \rightarrow D^{+} D^{-} \rightarrow D W^{+} D^{A} W^{-} \rightarrow D D W^{+} W^{-} Z, \quad \mathrm{e}^{+} \mathrm{e}^{-} \rightarrow D^{+} D^{-} \rightarrow D^{A} W^{+} D^{A} W^{-} \rightarrow D D W^{+} W^{-} Z Z$. As a result, the signature of the processes $\mathrm{e}^{+} \mathrm{e}^{-} \rightarrow D^{+} D^{-}$in the modes suitable for observation contains both (6) and processes with decay $W$ 's or $Z$ 's in the mentioned cascades:

$$
4 \div 1 \text { dijets and } 0 \div 5 \text { leptons with large } E_{T} \text { and large } M\left(E_{T}\right)+\text { nothing. }
$$

Note that the processes with invisible decay $Z \rightarrow v \bar{\nu}$ (we denote these states as $Z_{n}$, their $B R=20 \%$ ) have signature (6).

\section{2. $W$ Energy Distribution in $\mathrm{e}^{+} \mathrm{e}^{-} \rightarrow D^{+} D^{-} \rightarrow D D W^{+} W^{-}$}

Here we consider the energy distribution of $W$ with an effective mass $M^{*}$. At each value of $M^{*}$, we have in the rest frame of $D^{ \pm}$a two-particle decay $D^{ \pm} \rightarrow D W^{ \pm}$with $^{2}$

$$
\begin{aligned}
& E_{W}^{r}\left(M^{*}\right)=\frac{M_{+}^{2}+M^{* 2}-M_{D}^{2}}{2 M_{+}}, p_{W}^{r}\left(M^{*}\right)=\frac{\Delta\left(M_{+}^{2}, M^{* 2}, M_{D}^{2}\right)}{2 M_{+}}, \\
& \Delta\left(s_{1}, s_{2}, s_{3}\right)^{2}=s_{1}^{2}+s_{2}^{2}+s_{3}^{2}-2 s_{1} s_{2}-2 s_{1} s_{3}-2 s_{2} s_{3} .
\end{aligned}
$$

Denoting by $\theta$ the $W^{+}$escape angle in the $D^{+}$rest frame with respect to the direction of $D^{+}$motion in the laboratory frame and using $c \equiv \cos \theta$, we find the energy of $W^{+}$in the laboratory frame as $E_{W}^{L}=\gamma_{ \pm}\left(E_{W}^{r}+c \beta_{ \pm} p_{W}^{r}\right)$. Therefore, at given $M^{*}$, the energy $E_{W}^{L}$ of $W$ lies within the interval $\gamma_{ \pm}\left(E_{W}^{r} \pm \beta_{ \pm} p_{W}^{r}\right)$.

In particular, at $\boldsymbol{M}_{+}-\boldsymbol{M}_{\boldsymbol{D}}>\boldsymbol{M}_{W}$ we have $\boldsymbol{M}^{*}=M_{W}$, and the kinematical edges of the $W$ energy distribution are

$$
E_{W, \text { on }}^{L, \pm}=\gamma_{ \pm}\left(E_{W}^{r}\left(M_{W}\right) \pm \beta_{ \pm} p_{W}^{r}\left(M_{W}\right)\right) .
$$

At $\boldsymbol{M}_{+}-\boldsymbol{M}_{\boldsymbol{D}}<\boldsymbol{M}_{W}$ we have $0 \leq M^{*} \leq M_{+}-M_{D}$, and obtain similar edges, which are different for each value of $M^{*}$. The absolute upper and lower bounds on the energy distribution of $W$ are attained at $M^{*}=0$, they are equal to

$$
E_{W, \text { off }}^{L, \pm}=E\left(1 \pm \beta_{ \pm}\right)\left(1-M_{D}^{2} / M_{+}^{2}\right) / 2 \text {. }
$$

At the highest value $M^{*}=M_{+}-M_{D}$ we have $p_{W}^{r}=0$, and an interval (9) is reduced to a point, where the entire $W$ energy distribution has a maximum (peak) of

$$
\left.E_{W, p}^{L} \equiv E_{W}^{L, \pm}\right|_{\left(M^{*}=M_{+}-M_{D}\right)}=E\left(1-M_{D} / M_{+}\right) \text {. }
$$

\subsection{Single Lepton Energy Distribution in $\mathrm{e}^{+} \mathrm{e}^{-} \rightarrow D^{+} D^{-} \rightarrow D D W^{+} W^{-} \rightarrow D D q \bar{q} \ell v(6 b)$}

The fraction of such events for each separate lepton, $\mathrm{e}^{+}, \mathrm{e}^{-}, \mu^{+}$or $\mu^{-}$, is about 0.08 , their sum is about 0.33 of the total cross section of the process. We will speak, for definiteness, $\ell=\mu^{-}$and neglect the muon mass.

${ }^{2}$ We denote quantities in the rest system of $D^{ \pm}$and in the Lab system by using superscripts $r$ and $L$ respectively, additional superscript + or - corresponds to upper or lower value of this quantity. Subscripts on or off correspond to on shell or off shell $W$ 's, subscripts $p$ or $k$ mark values, correspondent to peak or kink. Other subscripts and superscripts look evident. 
Note that in the laboratory frame, for a $W$ with some energy $E_{W}^{L}$, its $\gamma$-factor and the velocity are $\gamma_{W L}=E_{W}^{L} / M^{*}$ and $\beta_{W L} \equiv \sqrt{1-\gamma_{W L}^{-2}}$.

We study the distribution ${ }^{3}$ of muons over its energy $\varepsilon, \mathrm{d} \sigma^{\mu}\left(\varepsilon \mid M_{+}, M_{D}\right) / \mathrm{d} \varepsilon$. We show that this distribution has singular points, whose positions are kinematically determined, i.e. model independent.

a) If $M_{+}-M_{D}>M_{W}$ we have $M^{*}=M_{W}$, and the muon energy and momentum in the rest frame of $W$ are $M_{W} / 2$. Just as above, denoting by $\theta_{1}$ the escape angle of $\mu$ relative to the direction of the $W$ in the laboratory frame and using $c_{1}=\cos \theta_{1}$, we find that the muon energy in the laboratory frame is $\varepsilon=\gamma_{W L}\left(1+c_{1} \beta_{W L}\right)\left(M_{W} / 2\right)$. Therefore, for these muons $\varepsilon^{+}\left(E_{W}^{L}\right) \geq \varepsilon \geq \varepsilon^{-}\left(E_{W}^{L}\right)$ where

$$
\varepsilon^{+}\left(E_{W}^{L}\right)=E_{W}^{L}\left(1+\beta_{W L}\right) / 2=E_{W}^{L}+\sqrt{\left(E_{W}^{L}\right)^{2}-M_{W}^{2}} / 2 \text { and } \varepsilon^{-}\left(E_{W}^{L}\right)=M_{W}^{2} /\left(4 \varepsilon^{+}\left(E_{W}^{L}\right)\right) .
$$

It is easy to check that the interval corresponding to energy $E_{1 W}^{L}<E_{W}^{L}$ is located entirely within the interval, correspondent to energy $E_{W}^{L}$. Therefore, all muon energies lie within the interval determined by the highest value of $W$ energy:

$$
\varepsilon^{+} \geq \varepsilon \geq \varepsilon^{-} \equiv \frac{M_{W}^{2}}{4 \varepsilon^{+}} \text {, where } \varepsilon^{+} \equiv \varepsilon^{+}\left(E_{W, o n}^{L,+}\right)=\frac{E_{W, o n}^{L,+}+\sqrt{\left(E_{W, o n}^{L,+}\right)^{2}-M_{W}^{2}}}{2} .
$$

(Note that $E_{W, o n}^{L,+}=\varepsilon^{+}+M_{W}^{2} /\left(4 \varepsilon^{+}\right)$.)

With a shift of $\varepsilon$ from these boundaries inwards, the density of states in the $\varepsilon$ distribution grows monotonically due to contributions of smaller values $E_{W}^{L}$ up to values $\varepsilon_{k}^{ \pm}$, corresponding to the lowest value of $W$ energy $E_{W, \text { on }}^{L,-}$ :

$$
\varepsilon_{k}^{-} \equiv \varepsilon^{-}\left(E_{W, \text { on }}^{L,-}\right)=\frac{E_{W, \text { on }}^{L,-}-\sqrt{\left(E_{W, \text { on }}^{L,-}\right)^{2}-M_{W}^{2}}}{2}, \quad \varepsilon_{k}^{+} \equiv \varepsilon^{+}\left(E_{W, \text { on }}^{L,-}\right)=\frac{M_{W}^{2}}{4 \varepsilon_{k}^{-}} .
$$

In these points the energy distributions of muons has kinks. Between these kinks, the $\varepsilon$-distribution is approximately flat.

Figure 1, the left plot, shows the energy distribution of muons for the case of the matrix element independent of $\theta_{1}$. Since positions of kinks are kinematically determined, it is not surprising that calculations for distinct models (containing different angular dependence) demonstrate variations in shapes but do not perturb the position of kinks.

b) If $M_{+}-M_{D}<M_{W}$, the $D^{ \pm}$decays to $D W^{* \pm}$, where $W^{* \pm}$ is an off-shell $W$ with an effective mass $M^{*} \leq M_{+}-M_{D}$. The calculations for each $M^{*}$ similar to shown above demonstrate that the muon energies are within the interval appearing at $M^{*}=0$ :

$$
\left(\varepsilon^{-}=0 ; \varepsilon^{+}=E_{W, o f f}^{L,+}\right) .
$$

Similarly to the preceding discussion, the increase of $M^{*}$ shifts the interval boundaries inwards. Therefore, the muon energy distribution increases monotonously from the outer bounds up to the maximum (peak) at $M^{*}=M_{+}-M_{D}$ (cf. (11)):

$$
\varepsilon_{p}=E\left(1+\beta_{ \pm}\right)\left(1-M_{D} / M_{+}\right) / 2 .
$$

To get an idea about the shape of the peak, we use the distribution of $W^{*}$ 's (dijets or $\ell v$ pairs) over the effective masses $M^{*}$ which is given by the spin-dependent factor $R_{s_{D}} p^{*} d M^{* 2}$ :

$$
R_{0}=\frac{p^{* 2}}{\left(M_{W}^{2}-M^{* 2}\right)^{2}}, R_{1 / 2}=\frac{\left(M_{+}^{2}+M_{D}^{2}-M^{* 2}\right)\left(2 M_{W}^{2}+M_{+}^{2}+M_{D}^{2}\right)-4 M_{+}^{2} M_{D}^{2}}{\left(M_{W}^{2}-M^{* 2}\right)^{2} M_{W}^{2}} .
$$

The density of muon states in energy is calculated by convolution of kinematically determined distribution with distribution (16). Neglecting the angular dependence of the matrix element, we obtain the result in form of

${ }^{3}$ We find useful, to mark in the argument of this distribution also masses of produced $D$-particle $M_{+}$and $D$-particle appeared in its decay $M_{D}$. 

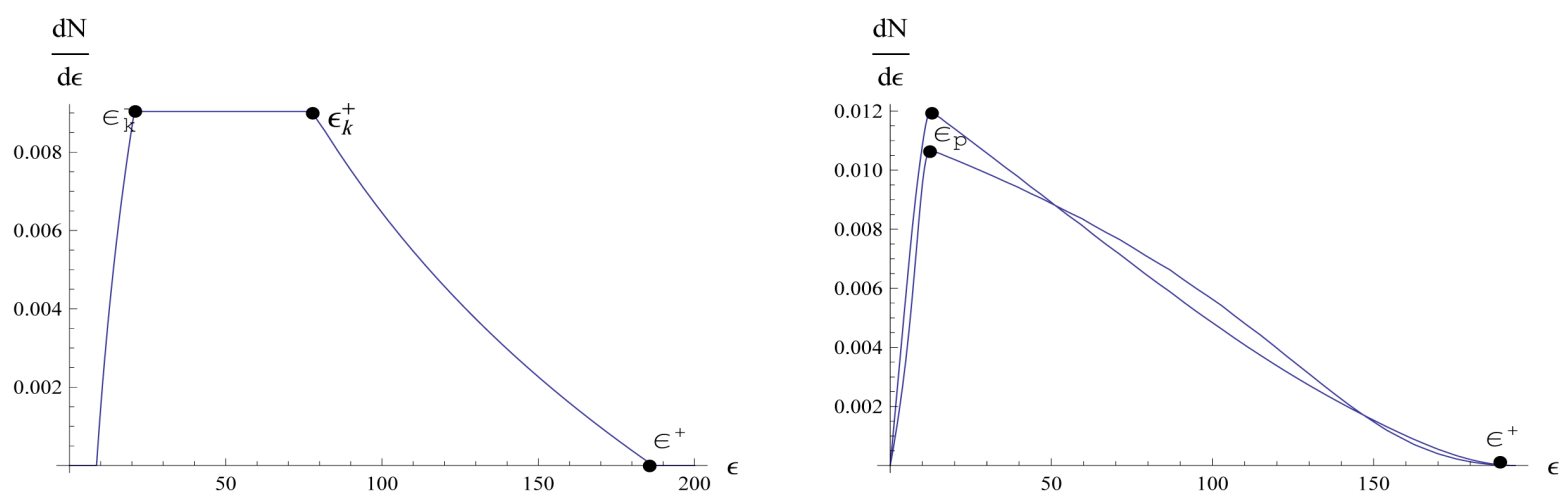

Figure 1. Distributions $\mathrm{d} N / \mathrm{d} \varepsilon \equiv(1 / \sigma) \mathrm{d} \sigma / \mathrm{d} \varepsilon$ at $E=250 \mathrm{GeV}, M_{D}=50 \mathrm{GeV}$ for $M_{+}=150 \mathrm{GeV}$-the case with $M_{+}-M_{D}>M_{W}$ (the right plot) and for $M_{+}=120 \mathrm{GeV}$-the case with $M_{+}-M_{D}<M_{W}$ (the left plot). In the latter case, the higher and lower peaks are for $s_{D}=0$ and $s_{D}=1 / 2$, respectively.

Figure 1, right plot. One can see that the discussed peak is sharp enough for both values of spin $s_{D}=0$ and $1 / 2$.

Characteristic values for singular points in the energy distributions of muons (kink and peak) together with similar points for the energy distributions of $W$ (dijets) are given in Table 1.

The cascade $D^{-} \rightarrow D W^{-} \rightarrow D \tau^{-} v \rightarrow D \mu^{-} v v v$ modifies the spectra just discussed. The energy distribution of $\tau$ produced in the decay $W \rightarrow \tau v$ is the same as that for $\mu$ or e, discussed above (within the accuracy of $\sim M_{\tau} / M^{*}$ ). Once produced, $\tau$ decays to $\mu v v$ in $17 \%$ of cases (the same for decay to e $v v$ ). These muons are added to those discussed above.

In the $\tau$ rest frame, the energy of muon is $E_{\mu}^{\tau}=y M_{\tau} / 2$ with $y \leq 1$. The energy spectrum of muons is $\mathrm{d} N / \mathrm{d} y=2(3-2 y) y^{2}$ (see textbooks). This spectrum and the distributions obtained above are converted into the energy distribution of muons in the Lab frame. It is clear that this contribution is strongly shifted towards the soft end of the entire muon energy spectrum.

The resulting distribution retains the upper boundary of the energy distribution of muons $\varepsilon^{+}$(12), (14). Numerical examples show that here the upper kink is smeared, while lower kink $\varepsilon_{k}^{-}$become even more sharp without shift from position (13) in wide region of masses $M_{+}$and $M_{D}$. The position of peak (15) is also shifted weakly.

\subsection{Additional Decay Channels at $M_{+}>M_{A}$}

At $M_{+}>M_{A}$ the decay $D^{ \pm} \rightarrow D^{A} W^{ \pm} \rightarrow D Z W^{ \pm}$become possible and the processes

$\mathrm{e}^{+} \mathrm{e}^{-} \rightarrow D^{+} D^{-} \rightarrow W^{+} W^{-} D^{A} D \rightarrow D D W^{+} W^{-} Z$, etc. with signature (7) should be taken into account.

The total probability of $D^{+}$decay to $D W^{+}$and $D^{A} W^{+}$equals 1 . The decay $D^{ \pm} \rightarrow D^{A} W^{ \pm}$is described by the same equation as $D^{ \pm} \rightarrow D W^{ \pm}$, but with other kinematical factors since $M_{A} \neq M_{D}$. In the IDM the probability of this new decay is lower than that without $D^{A}$ due to smaller final phase space, i.e.

$B=B R\left(D^{+} \rightarrow D^{A} W^{+}\right)<0.5$. In the MSSM value of $B$ depends additionally on the mixing angles. We assume that in general case $B \lesssim 0.5$.

Below we limit ourself by the study of processes with signature (6b), (7a). Unfortunately, some of new processes with intermediate $D^{A}$ look as those with signature (6) since large fraction (20\%) of decays of $Z$ is invisible ( $v \bar{v}$ final states). We denote these states of $Z$ as $Z_{n}$.

Let us consider in more detail production of an observed state with signature (6b), (7a) $\mu^{-}+(1-2)$ dijets + nothing. This state can be obtained from two different cascades.

1) The cascade $D^{-} \rightarrow D W^{-} \rightarrow D \mu^{-} v$. The energy distribution of $\mu^{-}$here reproduces $\mathrm{d} \sigma^{\mu}\left(\varepsilon \mid M_{+}, M_{D}\right) / \mathrm{d} \varepsilon$, discussed in Section 2.3 with an additional factor $(1-B)$.

2) Cascade $D^{-} \rightarrow D^{A} W^{-} \rightarrow D Z_{n} \mu^{-} v$. Since couplings $D^{-} D W^{-}$and $D^{-} D^{A} W^{-}$differ by a phase factor only (and perhaps mixing angle factors), the energy distribution of $\mu^{-}$in this case is described by the same dependence $\mathrm{d} \sigma$ but with the change $M_{D} \rightarrow M_{A}$, the corresponding contribution to the entire energy distribution is $0.2 B \mathrm{~d} \sigma^{\mu}\left(\varepsilon \mid M_{+}, M_{A}\right) / \mathrm{d} \varepsilon$. For brevity we will write $\mathrm{d} \sigma^{\mu}\left(\varepsilon \mid M_{+}, M_{D}\right) \rightarrow \mathrm{d} \sigma_{W}^{\mu}$ and 
Table 1. The singular point energies of lepton and dijet in $\mathrm{e}^{+} \mathrm{e}^{-} \rightarrow D^{+} D^{-} \rightarrow D D q \bar{q} \ell v$ (in $\mathrm{GeV}$ ) at $M_{D}=50 \mathrm{GeV}$.

\begin{tabular}{cccccccc}
\hline$E$ & $M_{+}$ & $\varepsilon^{+}$ & $\varepsilon_{k}^{-}$ & $\varepsilon_{k}^{+}$ & $\varepsilon_{p}$ & $E_{w_{p}}^{L}$ & $E_{w}^{L,+}$ \\
\hline 250 & 150 & 186.3 & 20.8 & 77.8 & - & - & 195.4 \\
250 & 200 & 184.9 & 34.9 & 46.3 & - & - & 193.6 \\
250 & 80 & 148.3 & - & - & 91.3 & 93.8 & 148.3 \\
100 & 80 & 78 & - & - & 30 & 37.5 & 78 \\
\hline
\end{tabular}

$\mathrm{d} \sigma^{\mu}\left(\varepsilon \mid M_{+}, M_{A}\right) \rightarrow \mathrm{d} \sigma_{W Z n}^{\mu}$. The resulting energy distribution is

$$
\mathrm{d} \sigma_{\text {tot }}^{\mu} / \mathrm{d} \varepsilon=(1-B) \mathrm{d} \sigma_{W}^{\mu} / \mathrm{d} \varepsilon+0.2 B \mathrm{~d} \sigma_{W Z n}^{\mu} / \mathrm{d} \varepsilon .
$$

The shape of the distribution $\mathrm{d} \sigma_{W Z n}^{\mu} / \mathrm{d} \varepsilon$ is similar to that for $\mathrm{d} \sigma_{W}^{\mu} / \mathrm{d} \varepsilon$, but with different positions of kinks and (or) peak. As $M_{A}>M_{D}$, these new kinks and (or) peak are situated below similar points for $\mathrm{d} \sigma_{W}^{\mu} / \mathrm{d} \varepsilon$. Since this contribution is much smaller than the main contribution $\mathrm{d} \sigma_{W}^{\mu} / \mathrm{d} \varepsilon$ (with the overall ratio $0.2 B /(1-B)$ at $B<0.5)$, it only results in a weak reshaping of the full energy distribution as compared with distributions $\mathrm{d} \sigma_{W}^{\mu} / \mathrm{d} \varepsilon$.

Note that in the case $M_{A} \approx M_{D}$ the distributions $\mathrm{d} \sigma_{W Z n}^{\mu} / \mathrm{d} \varepsilon$ and $\mathrm{d} \sigma_{W}^{\mu} / \mathrm{d} \varepsilon$ are close to each other, and $\mathrm{d} \sigma_{\text {tot }}^{\mu} / \mathrm{d} \varepsilon \propto \mathrm{d} \sigma_{W}^{\mu} / \mathrm{d} \varepsilon$. In the opposite degenerate case $M_{+} \approx M_{A}$, the quantity $B \ll 1$ and the influence of the intermediate $D^{A}$ state on the result is negligible. (Such very cases are widely discussed in context of MSSM).

\section{The Overall Picture}

Observation of events with signature (6), (7) will be a clear signal for DM particle candidates. The nonobservation of such events will allow to find lower limits for masses $M_{+}$, like [11]-[13]. One can hope that these limits will be close to the beam energy $E$.

At $M_{+}<E$, the cross section $\mathrm{e}^{+} \mathrm{e}^{-} \rightarrow D^{+} D^{-}$is a large fraction of the total cross section of $\mathrm{e}^{+} \mathrm{e}^{-}$ annihilation, and it makes this observation a very realistic task.

\subsection{Distortion of the Obtained Results}

A more detailed analysis reveals two sources of distortion of the obtained results (we neglect them in our preliminary analysis).

1. The final width of $W$ and $D^{ \pm}$( $Z$ and $D^{A}$ ) leads to a blurring singularities derived. This effect increases with the growth of $M_{+}-M_{D}$.

2. The energy spectra under discussion will be smoothed due to QED initial state radiation (ISR), final state radiation (FSR) and beamsstrahlung (BS). The ISR and FSR spectra are machine independent, while BS spectrum is specific for each machine (but well known during operations). This smoothing decreases accuracy in measuring of masses. However, the precise knowledge of mentioned spectra allows to solve the problem about restoration original accuracy by means methods of deconvolution in so called "incorrect inverse problem". This work and the estimates of the range where masses and spins can be determined with reasonable accuracy will be the subject of the forthcoming paper.

\subsection{Masses}

Masses $\boldsymbol{M}_{\boldsymbol{D}}$ and $\boldsymbol{M}_{+}$. In a well known approach, one measures edges in the energy distributions of dijets, representing $W$ in the decay $D^{ \pm} \rightarrow D W^{ \pm}$[5] [6]. However, the individual jet energies and consequently, effective masses of dijets cannot be measured with a high precision. The observed lower edge of the $W$ energy distribution in the dijet mode and the position of a peak in this distribution (11) are smeared by this uncertainty. One can only hope for a sufficiently accurate measurement of the upper edge of the $W$ energy distribution, $E_{W}^{L,+}$ (9), (10).

We suggest to extract the second quantity for description of masses from the lepton energy spectra. The 
lepton energy is measurable with a high accuracy. We found above that the singular points of the energy distribution of the leptons in the final state with signature (6a) are kinematically determined, and therefore can be used for a mass measurement.

M1) If a $D^{A}$ particle is absent or $M_{A}>M_{+}$, the results (12)-(15) describe the energy distributions completely. The shape of the energy distribution of leptons (with one peak or two kinks) allows to determine which case is realized, $M_{+}-M_{D}>M_{W}$ or $M_{+}-M_{D}<M_{W}$.

At $M_{+}-M_{D}>M_{W}$, the positions of upper edge in the dijet energy distribution $E_{W, o n}^{L,+}$ (9) and the lower kink in the muon energy distribution $\varepsilon_{k}^{+}$(13) give us two equation necessary for determination of $M_{D}$ and $M_{+}$. We reproduce these equations for clarity

$$
\begin{gathered}
E_{W, \text { on }}^{L, \pm}=\frac{E}{M_{+}}\left(E_{W}^{r} \pm \beta_{+} p_{W}^{r}\right), \varepsilon_{k}^{-}=\frac{E_{W, \text { on }}^{L,-}-\sqrt{\left(E_{W, \text { on }}^{L,-}\right)^{2}-M_{W}^{2}}}{2}, \text { where } \\
E_{W}^{r}=\frac{M_{+}^{2}+M_{W}^{2}-M_{D}^{2}}{2 M_{+}}, p_{W}^{r}=\frac{\Delta\left(M_{+}^{2}, M_{W}^{2}, M_{D}^{2}\right)}{2 M_{+}}, \beta_{+}=\sqrt{1-\frac{M_{+}^{2}}{E^{2}}} .
\end{gathered}
$$

At $M_{+}-M_{D}<M_{W}$, two similar equations are provided by the position of the upper edge in the dijet energy distribution $E_{W, \text { off }}^{L,+}(10)$ and the peak in muon energy distribution $\varepsilon_{p}$ (15).

In both cases the position of the upper edge in the dijet energy distribution $E_{W, \text { on }}^{L,+}$ or $E_{W, \text { off }}^{L,+}$ should be extracted from all events with signature (6), (7), the position of the lower kink in the muon energy distribution $\varepsilon_{k}^{+}$or peak $\varepsilon_{p}$ can be extracted from events with signature (6b) only.

M2) The signal of realization of the inequality $\boldsymbol{M}_{A}<M_{+}$will be observation of the process $\mathrm{e}^{+} \mathrm{e}^{-} \rightarrow D D^{A}$, having signature (26). In this case the position of the upper edge in the dijet energy distribution is the same as in previous case. The position of lower edge in the dijet energy distribution is either shifted or smeared, in this case the method of [5] [6] becomes completely inapplicable. The entire energy distribution of muons in the observed state $\mu+1$ or 2 dijet + nothing was described in the Section 2.4. It was shown there that taking into account a new decay channel $D^{-} \rightarrow D^{A} W^{-} \rightarrow D Z_{n} \mu^{-} v$ changes the position of the main singularities in the muon energy spectrum very weakly. Therefore the above mentioned procedure for finding $M_{+}$and $M_{D}$ can be used in this case as well.

The opportunity to extract new singularities from the data, related to $\mathrm{d} \sigma_{W Z n}^{\mu} / \mathrm{d} \varepsilon$ (and giving additionally $M_{A}$ ), requires a separate study (see also analysis in Appendix B).

\subsection{Spin of $D$-Particles $S_{D}$}

- The amplitude of the process $\mathrm{e}^{+} \mathrm{e}^{-} \rightarrow D^{+} D^{-}$is the sum of model-independent QED diagram (the photon annihilation), the $Z$ annihilation diagram and in some models $t$-channel exchange by other $D$-odd particles. We start with the description of cross section in the minimal approximation, taking into account only photon and $Z$ annihilation diagrams. Neglecting terms $\propto\left(1 / 4-\sin ^{2} \theta_{W}\right)$ (described $\gamma-Z$ interference) we have:

$$
\sigma_{\min }\left(s_{D}\right)=\sigma_{0} \begin{cases}\beta_{ \pm}\left[1+\frac{2 M_{+}^{2}}{s}+r_{Z} \beta_{ \pm}^{2}\right] & \left(s_{D}=\frac{1}{2}\right), \\ \beta_{ \pm}^{3}\left[\frac{1}{4}+r_{Z} \cos ^{2}\left(2 \theta_{W}\right)\right] & \left(s_{D}=0\right),\end{cases}
$$

where $r_{Z}=\frac{\mu_{M}}{\left(2 \sin \left(2 \theta_{W}\right)\right)^{4}\left(1-M_{Z}^{2} / s\right)^{2}}=\frac{0.124 \mu_{M}}{\left(1-M_{Z}^{2} / s\right)^{2}}$, factor $\mu_{M} \leq 1$ is expressed via parameters of possible mixing, etc. Figure 2 and Table 2 represent dependence of $\sigma_{\min } \mathrm{e}^{+} \mathrm{e}^{-} \rightarrow D^{+} D^{-} / \sigma_{0}$ (19) on beam energy $E=\sqrt{s} / 2$ for $\mu_{M}=1$.

The cross section of the process is reduced by contribution of the diagram with $t$-channel exchange by other $D$-odd particle $D_{F}$. This decrease is not so strong if mass of $D_{F}$ is high enough. For example, if mass of selectron is more than $250 \mathrm{GeV}$ (condition 2 in Section 1 and [20]), the cross section for $s_{D}=1 / 2$ is reduced by a factor $\geqslant 0.6, \sigma\left(s_{D}=1 / 2\right) \geq 0.6 \sigma_{\min }\left(s_{D}=1 / 2\right)$. Combining with numbers from Figure 2 and Table 2 we 


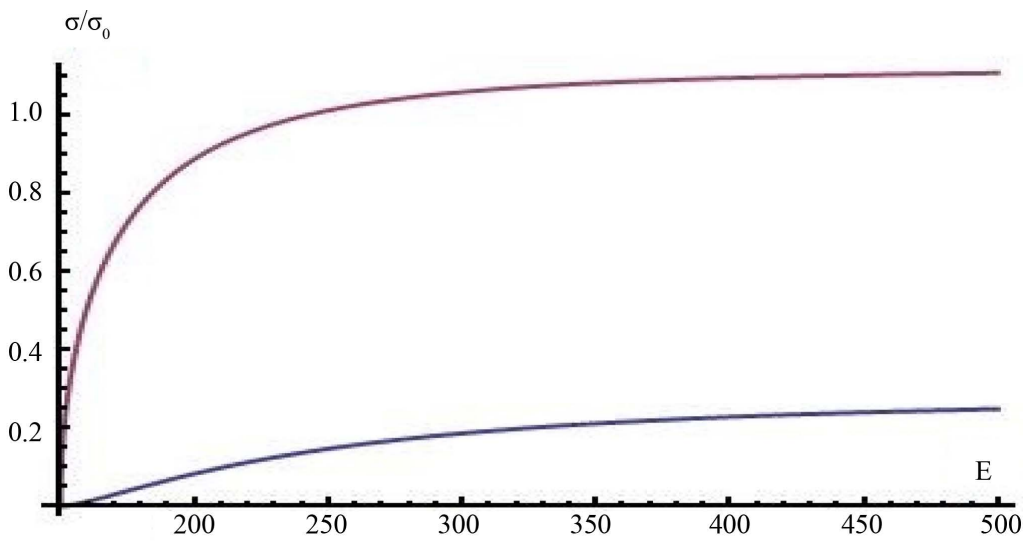

Figure 2. The upper curve for $s_{D}=1 / 2$, the lower for $s_{D}=0 ; M_{+}=150 \mathrm{GeV}$.

Table 2. Some values of $\sigma_{\min }\left(\mathrm{e}^{+} \mathrm{e}^{-} \rightarrow D^{+} D^{-}\right) / \sigma_{0}$.
\begin{tabular}{ccccc}
\hline$E, \mathrm{GeV}$ & 100 & 250 & & \\
\hline$M_{+}, \mathrm{GeV}$ & 80 & 80 & 150 & 250 \\
$S_{D}=0: \quad \sigma / \sigma_{0}$ & 0.066 & 0.245 & 0.162 & 0.062 \\
$s_{D}=1 / 2: \quad \sigma / \sigma_{0}$ & 0.84 & 1.107 & 1.02 & 0.82 \\
\hline
\end{tabular}

obtain (for identical masses $M_{+}$at a given beam energy $E$ ):

$$
\sigma\left(s_{D}=1 / 2\right) \geq 0.6 \sigma_{\min }\left(s_{D}=0\right)
$$

- The experimental value of the $\mathrm{e}^{+} \mathrm{e}^{-} \rightarrow D^{+} D^{-}$cross section is obtained by summing over all processes with signature (6), (7) (that is about 3/4 of the total cross section). By taking into account the known BR's for $W$ decay the accuracy of this restoration of $\sigma\left(\mathrm{e}^{+} \mathrm{e}^{-} \rightarrow D^{+} D^{-}\right)$can be improved.

When masses $M_{+}$become known, the cross section $\sigma_{\min }\left(\mathrm{e}^{+} \mathrm{e}^{-} \rightarrow D^{+} D^{-}\right)$is calculated with reasonable precision with Equation (19). The strong inequality (20) allows to determine spin $s_{D}$ from the obtained values of cross sections even with a handful of well-reconstructed events.

\section{Conclusions}

We consider models in which stability of dark matter particles $D$ is ensured by conservation of new quantum number referred to as $D$-parity. Besides these models contain charged particles $D^{ \pm}$with the same $D$-parity. (Examples Inert Doublet Model with scalar $D$-particles and MSSM with $D$-particle of spin $1 / 2$ and $D$-parity equal $R$-parity). In these models we have studied the energy distribution of single lepton in the process like $\mathrm{e}^{+} \mathrm{e}^{-} \rightarrow D^{+} D^{-} \rightarrow D D W^{ \pm}(\rightarrow q q) W^{\mp}(\rightarrow \ell v)$, having high enough cross section. Simple analysis allows us to establish that this distribution has singular points, kinks, peaks and end points, which are driven by kinematics only, and therefore are model-independent. Based on this analysis, we propose to use the mentioned distribution at future linear $\mathrm{e}^{+} \mathrm{e}^{-}$collider ILC, CLIC, etc. for precise measuring of masses of dark matter particles and charged particles $D^{ \pm}$.

This method is in several aspects superior to the standard approaches discussed elsewhere.

1) It uses leptons which are copious and can be accurately measured in contrast with jets that individual energy can be measured only with lower precision.

2) These singularities are robust and survive even when superimposed on top of any smooth background.

In addition, even a rough measurement of cross sections with a very clean signature allows us to determine spin of DM particles based on the results of mentioned kinematical measurements.

\section{Acknowledgments}

This work was supported in part by grants RFBR and NSh-3802.2012.2, Program of Dept. of Phys. Sc. RAS and 
SB RAS "Studies of Higgs boson and exotic particles at LHC” and Polish Ministry of Science and Higher Education Grant N202 230337. I am thankful to A. Bondar, E. Boos, A. Gladyshev, A. Grozin, S. Eidelman, I. Ivanov, D. Ivanov, D. Kazakov, J. Kalinowski, K. Kanishev, P. Krachkov and V. Serbo for discussions.

\section{References}

[1] Hooper, D. (2009) TASI 2008 Lectures on Dark Matter. arXiv:0901.4090 [hep-ph]

[2] Maniatis, M. (2009) The Next-to-Minimal Supersymmetric extension of the Standard Mod. arXiv:0906.0777 [hep-ph]

[3] Kazakov, D.I. (2010) Supersymmetry on the Run: LHC and Dark Matter. arXiv:1010.5419 [hep-ph]

[4] Ellis, J. (2010) New Light on Dark Matter from the LHC. arXiv:1011.0077 [hep-ph]

[5] Li, Y. and Domerotski, A. (2010) Chargino and Neutralino Masses at ILC. arXiv: 1007.0698

[6] Asano, M., Fujii, K., Hundi, R.S., Itoh, H., Matsumoto, S., Okada, N., Saito, T., Suehara, T., Takubo, Y. and Yamamoto, H. (2011) Identification of new physics and general WIMP search at the ILC. arXiv:1007.2636 [hep-ph]

[7] Deshpande, N.G. and Ma, E. (1978) Physical Review D, 18, 2574-2576. http://dx.doi.org/10.1103/PhysRevD.18.2574

[8] Barbieri, R., Hall, L.J. and Rychkov, V.S. (2006) Improved Naturalness with a Heavy Higgs: An Alternative Road to LHC Physics. arXiv:hep-ph/0603188.

[9] Ginzburg, I.F., Kanishev, K.A., Krawczyk, M. and Sokolowska, D. (2010) Evolution of Universe to the present inert phase. arXiv:1009.4593 [hep-ph]

[10] Gustafsson, M., Rydbeck, S., Lopez-Honorez, L. and Lundstrom, E. (2012) Physical Review D, 86, Article ID: 075019. http://dx.doi.org/10.1103/PhysRevD.86.075019

[11] Espirito Santo, M., Hultqvist, K., Johansson, P. and Lipniacka, A. (2003) DELPHI 2003-002 PHYS 928. http://delphiwww.cern.ch/pubxx/delnote/dn2003.html

[12] Lundstrom, E., Gustafsson, M. and Edsjo, J. (2009) Inert Doublet Model and LEP II Limits. arXiv:0810.3924 [hep-ph]

[13] Aoki, M., Kanemura, S. and Yokoya, H. (2013) Reconstruction of Inert Doublet Scalars at the International Linear Collider. arXiv:1303.6191 [hep-ph]

[14] Dolle, E.M. and Su, S. (2009) Physical Review D, 80, Article ID: 055012. http://dx.doi.org/10.1103/PhysRevD.80.055012

[15] Dolle E., Miao X., Su S., Thomas B. (2010) Physical Review D, 81, Article ID: 035003. http://dx.doi.org/10.1103/PhysRevD.81.035003

[16] Lopez Honorez, L., Nezri, E., Oliver, J.F. and Tytgat, M.H.G. (2007) The Inert Doublet Model: An Archetype for Dark Matter. arXiv:0612275 [hep-ph]

[17] Nezri, E., Tytgat, M.Y.G. and Vertongen, G. (2009) Positrons and antiprotons from inert doublet model dark matter. arXiv:0901.2556 [hep-ph]

[18] Andreas, S., Hambye, T. and Tytgat, M.H.G. (2008) WIMP Dark Matter, Higgs Exchange and DAMA. arXiv: 0808.0255 [hep-ph]

[19] Honorez, L.L. and Yaguna, C.E. (2010) The Inert Doublet Model of Dark Matter Revisited. arXiv:1003.3125 [hep-ph]

[20] Particle Data Group (2012) Physical Review D, 86, Article ID: 010001. http://dx.doi.org/10.1103/PhysRevD.86.010001

[21] Heuer, R.D., et al. (2001) TESLA Technical Design Report, DESY 2001-011, TESLA Report 2001-23, TESLA FEL 2001-05.

[22] International Linear Collider TDR (2013) The International Linear Collider Technical Design Report-Volume 2: Physics. arXiv:1306.6352 [hep-ph]

[23] Moortgat-Pick, G. (2008) Journal of Physics: Conference Series, 110, Article ID: 072027. http://dx.doi.org/10.1088/1742-6596/110/7/072027

[24] Freitas, A., Martyn, H.U., Nauenberg, U. and Zerwas, P.M. (2004) Sleptons: Masses, Mixings, Couplings. arXiv: hep$\mathrm{ph} / 0409129$

[25] Conley, J.A., Dreiner, H.K. and Wienemann, P. (2012) Measuring a Light Neutralino Mass at the ILC: Testing the MSSM Neutralino Cold Dark Matter Model. arXiv:1012.1035 [hep-ph]

[26] Ginzburg, I.F (2010) Simple and robust method for search Dark Matter particles and measuring their properties at ILC in various models of DM. arXiv:1010.5579 [hep-ph] 


\section{Appendix}

\section{A. Inert Doublet Model (IDM)}

The IDM the $Z_{2}$ symmetric Two Higgs Doublet Model, containing two scalar doublets $\phi_{S}$ and $\phi_{D}$, in which fermions are coupled to only field $\phi_{S}$. The $Z_{2}$ symmetry forbids $\phi_{S}, \phi_{D}$ mixing. It is described by the Lagrangian

$$
L=L_{g f}^{S M}+L_{Y}\left(\psi_{f}, \phi_{S}\right)+\left(D_{\mu} \phi_{S}^{\dagger} D_{\mu} \phi_{S}+D_{\mu} \phi_{D}^{\dagger} D_{\mu} \phi_{D}\right) / 2-V .
$$

Here, $L_{a f}^{S M}$ describes the $S U(2) \times U(1)$ Standard Model interaction of gauge bosons and fermions. The $L_{Y}$ describes the Yukawa interaction of fermions $\psi_{f}$ with only one scalar doublet $\phi_{S}$, having the same form as in the SM, $D_{\mu}$ is standard covariant derivative,

$$
\begin{aligned}
V= & -\frac{1}{2}\left[m_{11}^{2}\left(\phi_{S}^{3} \phi_{S}\right)+m_{22}\left(\phi_{D} \phi_{D}\right)\right]+\frac{\lambda_{1}}{2}\left(\phi_{S} \phi_{S}\right)^{2}+\frac{\lambda_{2}}{2}\left(\phi_{D} \phi_{D}\right)^{2}+\lambda_{3}\left(\phi_{S} \phi_{S}\right)\left(\phi_{D} \phi_{D}\right) \\
& +\lambda_{4}\left(\phi_{S}^{\dagger} \phi_{D}\right)\left(\phi_{D}^{\dagger} \phi_{S}\right)+\frac{\lambda_{5}}{2}\left[\left(\phi_{S}^{\dagger} \phi_{D}\right)^{2}+\left(\phi_{D}^{\dagger} \phi_{S}\right)^{2}\right] .
\end{aligned}
$$

All parameters of this Higgs potential are real and condition of its stability has form

$$
\lambda_{1}>0, \quad \lambda_{2}>0, \quad R=\frac{\lambda_{3}+\lambda_{4}+\lambda_{5}}{\sqrt{\lambda_{1} \lambda_{2}}}>-1
$$

The IDM is realized at

$$
\lambda_{5}<0, \quad \lambda_{4}+\lambda_{5}<0, \quad m_{11}^{2}>0, \begin{cases}\frac{m_{11}^{2}}{\sqrt{\lambda_{1}}}>\frac{m_{22}^{2}}{\sqrt{\lambda_{2}}} & \text { at } R>1 \\ R \frac{m_{11}^{2}}{\sqrt{\lambda_{1}}}>\frac{m_{22}^{2}}{\sqrt{\lambda_{2}}} & \text { at }|R|<1 .\end{cases}
$$

In this case $\left\langle\phi_{S}\right\rangle=\left(\begin{array}{l}0 \\ v\end{array}\right),\left\langle\phi_{D}\right\rangle=0$. Therefore, the doublet $\phi_{S}$ is responsible for electroweak symmetry breaking and the masses of fermions and gauge bosons just as in the Standard Model (SM). The second scalar doublet $\phi_{D}$ doesn't receive vacuum expectation value and doesn't couple to fermions.

Four degrees of freedom of the Higgs doublet $\phi_{S}$ are such as in the SM: three Goldstone modes become longitudinal components of the EW gauge bosons, one component becomes the standard Higgs boson $h$. All the components of the scalar doublet $\phi_{D}$ are realized as the massive $D$-particles: two charged $D^{ \pm}$and two neutral ones $D, D^{A}$ with masses $M_{+}, M_{D}, M_{A}$ respectively.

$$
M_{A}^{2}=M_{D}^{2}-\lambda_{5} v^{2}, \quad M_{+}^{2}=M_{D}^{2}-\left(\lambda_{4}+\lambda_{5}\right) v^{2} / 2 \text {.e }
$$

By construction, $D$-particles possess a new conserved multiplicative quantum number (named here as $D$ parity) and therefore the lightest particle among them can be a candidate for DM particle. In this model all $D$ particles have spin $s_{D}=0$. The other features of IDM can be found in ref. [7]-[10].

\section{B. Process $\mathrm{e}^{+} \mathrm{e}^{-} \rightarrow \mathrm{Z} \rightarrow D D^{A} \rightarrow D D Z$}

One more process leading to production of $D$-odd particles at ILC is also observable at $M_{A}+M_{D}<2 E$ (in particular, at $E>M_{+}>M_{A}$ ):

$$
\mathrm{e}^{+} \mathrm{e}^{-} \rightarrow Z \rightarrow D D^{A} \rightarrow D D Z \text {. }
$$

This process has a clear signature in the modes suitable for observation

The $\mathrm{e}^{+} \mathrm{e}^{-}$or $\mu^{+} \mu^{-}$pair with large $E_{T}$ and large $M\left(E_{T}\right)+$ nothing. The effective mass of this dilepton is $\leq M_{Z}$, its energy is typically less than $E$. 
A quark dijet with large $Z_{T}$ and large $M\left(Z_{T}\right)+$ nothing. The effective mass of this dijet is $\leq M_{Z}$, its energy is typically less than $E$.

At $M_{A}<M_{+}$the BR for channel with signature (26a) is 0.06, for the channel with signature (26b) 0.7 . We skip channel $Z \rightarrow \tau^{+} \tau^{-}$with $B R=0.03,20 \%$ of decays of $Z$ are invisible $(Z \rightarrow v \bar{v})$.

At $M_{A}>M_{+}$BR's for processes with signature (26) become less, since new decay channels $D^{A} \rightarrow D^{\mp} W^{ \pm} \rightarrow D W^{+} W^{-}$are added with signature

$$
\begin{aligned}
& \mathrm{e}^{+} \mathrm{e}^{-} \rightarrow D D^{A} \rightarrow D D W^{+} W^{-}: \text {Two quark dijets or dijet }+ \text { single lepton or } \\
& \text { two leptons in one hemisphere with large } Z_{T} \text { and large } M\left(Z_{T}\right)+\text { nothing. } \\
& \text { The effective mass of this system is } \leq M_{Z} \text {, its energy is typically less than } E \text {. }
\end{aligned}
$$

The cross section of the process $\mathrm{e}^{+} \mathrm{e}^{-} \rightarrow D D^{A}$ is model dependent. In the IDM it is determined unambiguously, in MSSM result depends on mixing angles and on the nature of fermions $D$ and $D^{A}$ (Dirac or Majorana). In all considered cases at $\sqrt{s}>200 \mathrm{GeV}$ this cross section is smaller than $0.1 \sigma_{0}$. Since the BR for events with signature (26a) is 0.06 , at the luminosity (3) annual number of events with this signature is smaller than $2 \times 10^{3}$. This number looks insufficient for kinematical analysis with high enough precision, (but limitations for masses can be obtained (cf. [11]-[13] for LEP)).

Nevertheless we describe, for completeness, the energy distributions of $Z$ in this process. The obtained equations are similar to (4), (8)-(10) for new kinematics.

The $\gamma$-factor and velocity of $D^{A}$ in c.m.s. for $\mathrm{e}^{+} \mathrm{e}^{-}$are

$$
\gamma_{A}=\frac{s+M_{A}^{2}-M_{D}^{2}}{4 E M_{A}}, \quad \beta_{A}=\frac{\Delta\left(s, M_{A}^{2}, M_{D}^{2}\right)}{s+M_{A}^{2}-M_{D}^{2}} .
$$

For production of $Z$ with an effective mass $M^{*}\left(M^{*}=M_{Z}\right.$ at $M_{A}-M_{D}>M_{Z}$ and $M^{*} \leq M_{A}-M_{D}$ at $M_{A}-M_{D}<M_{Z}$ ) in the rest frame of $D^{A}$

$$
E_{Z}^{r}=\frac{M_{A}^{2}+M^{* 2}-M_{D}^{2}}{2 M_{A}}, \quad p_{Z}^{r}=\frac{\Delta\left(M_{A}^{2}, M^{* 2}, M_{D}^{2}\right)}{2 M_{A}} .
$$

At $M_{A}-M_{D}>M_{Z}$ the $Z$-boson energy $E_{Z}^{L}$ lies within the interval with edges

$$
E_{Z, o n}^{L,-}=\gamma_{A}\left(E_{Z}^{r}-\beta_{A} p_{Z}^{r}\right), E_{Z, o n}^{L,+}=\gamma_{A}\left(E_{Z}^{r}+\beta_{A} p_{Z}^{r}\right) .
$$

At $M_{A}-M_{D}<M_{Z}$ similar equations are valid for each value of $M^{*}$. Absolute upper and lower edges of the energy distribution of $Z$ are reached at $M^{*}=0$ :

$$
E_{Z, \text { off }}^{L, \pm}=\gamma_{A}\left(1 \pm \beta_{a}\right)\left(M_{A}^{2}-M_{D}^{2}\right) /\left(2 M_{A}\right) \text {. }
$$

The peak in the energy distribution of dilepton appears at $M^{*}=M_{A}-M_{D}$ :

$$
E_{Z, p}^{L}=\gamma_{A}\left(M_{A}-M_{D}\right) \text {. }
$$

Masses $\boldsymbol{M}_{\boldsymbol{D}}$ and $\boldsymbol{M}_{A}$. At first sight, measurement of kinematical edges of the dilepton spectrum (30) (at $M_{A}-M_{D}>M_{Z}$ ) gives two equations for $M_{D}$ and $M_{A}$, allowing for determination of these masses. At $M_{A}-M_{D}<M_{Z}$, the same procedure can be performed separately for each value of the effective mass of dilepton [26]. In the latter case, the absolute edges of the dilepton energy spectrum (31) and the position of the peak in this spectrum (32) could be also used for measuring $M_{D}$ and $M_{A}$.

In any case, the upper edge in the dijet energy spectrum $E_{Z}^{L_{+}+}$(30), (31) (signature (26)) gives one equation, necessary to find $M_{A}$ and $M_{D}$. In principle, necessary additional information gives position of lower edge in the dilepton energy spectrum $E_{Z}^{L,-}$. However, as it was noted above, the anticipated number of events with signature (26a) looks insufficient for obtaining precise results. Together with good results for $M_{D}$ and $M_{+}$, one can hope to find an accurate value of $M_{A}$. 


\section{Backgrounds}

\section{C1. Background to the process with signature (6)}

We show here that the cross sections of possible background processes (with suitable simple cuts) are $\sim 10 \div 100$ times less than the cross section of the signal process and therefore they can be neglected at analysis. Note that some our estimates can be corrected due to ISR and beamstrahlung.

$B W 1$. The process $\mathrm{e}^{+} \mathrm{e}^{-} \rightarrow W^{+} W^{-}$gives the same final state as those with signature (6). However, many of its features are not permitted by this signature. This fact allow to exclude the BW1 process from analysis with a good confidence applying suitable cuts.

Let us discuss e.g. the observable mode $\mu^{-}+j j+Z_{T}$.

(a) For the process BW1 energy of each dijet $E_{i j}=E$.

Application of cut in the dijet energy $E_{j j}<E^{c^{j}}$ with large enough $E-E^{c}$ keeps all dijets from the signal process and leaves only small fraction of cross section of process BW1.

The dijet energy $E_{j j}$ in BW1 can be less than $E^{c}$ only if $W^{-}$(seen as $\ell^{-} v$ ) is strongly off shell with effective mass much higher than $M_{W}$. The probability of such situation is estimated easily, it is $\delta_{W 1 a} \approx \Gamma_{w} M_{W} /\left(\pi E\left(E-E^{c}\right)\right)$. The examples considered in the Table 1 allow to use cut $E^{c}=200 \mathrm{GeV}$ and this cut leaves only 0.0012 of total cross section of BW1 process.

(b) For BW1 the missing mass $M\left(E_{T}\right)=0$. Application of cut $M\left(Z_{T}\right)>M^{c}$ with suitable $M^{c}$ keeps all events of the signal process but diminishes contribution of BW1 in the events with signature (6A) even further.

For dijet+dijet mode total energy of these dijets in the process BW1 differs from $2 E$ only due to inaccuracy of measurements. For the examples considered in the Table 1 this energy deficit should be larger than $100 \mathrm{GeV}$.

$B W 2$. The same (in its content) final state as we consider for signal process can be achieved via mechanism without at least one intermediate $D^{ \pm}$in $s$-channel, e.g.

$\mathrm{e}^{+} \mathrm{e}^{-} \rightarrow\left(W^{-} \rightarrow \mu^{-} \bar{v}\right)\left(W^{+} \rightarrow D\left(D^{+} \rightarrow D W^{+} \rightarrow D q \bar{q}\right)\right)$. To simplify text of discussion, we will write here about the case $M_{A}>M_{+}$only.

The contribution of this mechanism to the total cross section is at least in $\alpha$ times less then that of the signal process. Indeed, in the signal process the value of cross section is given by the second order process

$\mathrm{e}^{+} \mathrm{e}^{-} \rightarrow D^{+} D^{-}$. It includes the intermediate decay $D^{+} \rightarrow D W^{+}$with probability 1 , the corresponding cross section is $\sim \alpha / s$ (an additional $\alpha$ in the formal diagram is compensated by the small $D^{+}$width $\Gamma^{+}$in the denominator of propagator). In the process BW2 we have third order process with decays in final stage (if $M_{+}-M_{D}<M_{W}$ that is even the fourth order process). The neutrino exchange term enhances this contribution only logarithmically. If necessary, it can be reduced by variation of longitudinal polarization of initial electron and additionally by the cut in transverse momentum of muon $p_{\mu \perp}>40 \mathrm{GeV}$.

The interference of this BW2 mechanism with the signal one is also very small. In particular, in the signal process final leptons $\left(\ell^{-} v\right)$ and $d$ form system with effective mass $M_{+} \pm\left(\sim \Gamma^{+}\right)$, while in the process BW2 this value of effective mass is only small part of entire phase space of this system, contributed to the total cross section.

Therefore, the contribution of mechanism BW2 can be neglected with accuracy better than $1 \%$.

$B W 3$. $\mathrm{e}^{+} \mathrm{e}^{-} \rightarrow D D^{A} \rightarrow D D^{+} W^{-} \rightarrow D D W^{+} W^{-}$. This background is absent if $M_{A}<M_{+}$or $M_{A}+M_{D}>\sqrt{s}$. If $\sigma\left(\mathrm{e}^{+} \mathrm{e}^{-} \rightarrow D D^{A}\right)$ is not small at given $\sqrt{s}$, this fact will be seen via an observation of the process $\mathrm{e}^{+} \mathrm{e}^{-} \rightarrow D D Z$ (26). The cross section $\sigma(B W 3)<\sigma\left(\mathrm{e}^{+} \mathrm{e}^{-} \rightarrow D D Z\right)$, i.e. it is much less than $\sigma\left(\mathrm{e}^{+} \mathrm{e}^{-} \rightarrow D^{+} D^{-} \rightarrow D D W^{+} W^{-}\right)$(roughly, by one order of magnitude). In this process all recorded particles move in one hemisphere in contrast to the with signal process, where they move in two opposite hemispheres. Therefore, the contribution of this background process may be reduced additionally by application of suitable cuts.

$B W 4$. In the SM processes with an observed state, satisfying criterion (6), large $Z_{T}$ is carried away by additional neutrinos. The corresponding cross section is at least one electroweak coupling constant squared $g^{2} / 4 \pi$ or $g^{\prime 2} / 4 \pi$ smaller than $\sigma_{0}$, with $g^{2} / 4 \pi \sim g^{\prime 2} / 4 \pi \sim \alpha$. Therefore, $\sigma(B W 3) \lesssim 0.01 \sigma\left(\mathrm{e}^{+} \mathrm{e}^{-} \rightarrow D^{+} D^{-}\right)$.

\section{C2. Background to $\mathrm{e}^{+} \mathrm{e}^{-} \rightarrow D D^{A} \rightarrow D D \ell^{+} \ell^{-}$}

We consider these background processes only for completeness, since anticipated number of events for the process itself is not so large. We subdivide these background processes into 3 groups.

BZ1. $\mathrm{e}^{+} \mathrm{e}^{-} \rightarrow Z Z_{n}$. At first sight, this process can mimic the process $\mathrm{e}^{+} \mathrm{e}^{-} \rightarrow D D Z$. However, the dilepton 
or dijet in this process have the same energy $E$ as the colliding electrons. The criterion (26) excludes such events from the analysis.

The cross section $\sigma\left(\mathrm{e}^{+} \mathrm{e}^{-} \rightarrow Z Z_{n}\right) \sim 0.2 \times 3 r_{Z} \sigma_{0} \ln \left(s / M_{Z}^{2}\right)$. The variants of this process with off-shell $Z$, giving another effective mass of observed dijet or dilepton but with energy close to $E$, has cross section which is smaller by a factor $\sim \alpha$.

BZ2. Processes with independent production of separate $\ell_{i}$ :

(BZ2.1) $\mathrm{e}^{+} \mathrm{e}^{-} \rightarrow D D Z \rightarrow D D \tau^{+} \tau^{-} \rightarrow D D \ell_{1}^{+} \ell_{2}^{-}+v^{\prime} s$,

(BZ2.2) $\mathrm{e}^{+} \mathrm{e}^{-} \rightarrow D D^{A} \rightarrow D D W^{+} W^{-} \rightarrow D D \ell_{1} \ell_{2} v \bar{v}$,

(BZ2.3) $\mathrm{e}^{+} \mathrm{e}^{-} \rightarrow D^{+} D^{-} \rightarrow D D W W \rightarrow D D \ell_{1} \bar{\ell}_{2} v \bar{v}$

(BZ2.4) $\mathrm{e}^{+} \mathrm{e}^{-} \rightarrow W^{+} W^{-} \rightarrow \ell_{1} \bar{\ell}_{2} v \bar{v}$.

In these processes $\mathrm{e}^{+} \mathrm{e}^{-}, \mu^{+} \mu^{-}, \mathrm{e}^{-} \mu^{+}$and $\mathrm{e}^{+} \mu^{-}$pairs are produced with identical probability and identical distributions. Hence, the subtraction from the $\mathrm{e}^{+} \mathrm{e}^{-}$and $\mu^{+} \mu^{-}$data the measured distributions of $\mathrm{e}^{-} \mu^{+}$and $\mathrm{e}^{+} \mu^{-}$eliminates a contribution of these processes from the energy distributions under interest. This procedure does not implement substantial inaccuracies since cross sections of these processes after suitable cuts are small enough.

The cross sections of processes (BZ2.1), (BZ2.2) are small in comparison with that for

$\mathrm{e}^{+} \mathrm{e}^{-} \rightarrow \rightarrow D D Z \rightarrow D D \mu^{+} \mu^{-}$. In the process (BZ2.3) leptons are flying in the opposite hemisphere, in contrast to the process under study $\mathrm{e}^{+} \mathrm{e}^{-} \rightarrow D D Z \rightarrow D D \mu^{+} \mu^{-}$, where the leptons are flying in the same hemisphere. The cross section of the process (BZ2.4) is basically large. The application of cuts $E_{\bar{\ell} \bar{\ell}}<E, \quad M_{\bar{\ell} \bar{\ell}} \leq M_{Z}$ leaves less

than $\left(M_{Z}^{2} / s\right)^{2} \ln \left(s / M_{Z}^{2}\right)$ part of the cross section. The obtained quantity becomes smaller than that for the signal.

BZ3. In the SM processes with the observed state (26), the large $E_{T}$ is carried away by additional neutrino(s). The magnitude of the corresponding cross sections is at least by one electroweak coupling constant squared $g^{2} / 4 \pi$ or $g^{\prime 2} / 4 \pi$ less than $\sigma_{0}$, with $g^{2} / 4 \pi \sim g^{\prime 2} / 4 \pi \sim \alpha$. Therefore, the cross sections of these processes are at least one order of magnitude smaller than the cross section for this signal process. 